\title{
Vancomycin-resistant enterococci in Canada revisited
}

\author{
B Lynn Johnston MD FRCPC ${ }^{1}$, John M Conly MD FRCPC ${ }^{2}$
}

$\mathrm{I}$ n the July/August 1997 issue of the The Canadian Journal of Infectious Diseases, the Adult Infectious Disease Notes was entitled "The emerging epidemiology of vancomycin-resistant enterococci (VRE) in Canada"(1). At that time, it was acknowledged that VRE's Canadian epidemiology was not fully elucidated. The first isolate of VRE had been reported in Edmonton in 1993, and the first outbreak of VRE in Canada occurred in Toronto in 1995 (1). This outbreak was presented as "A casecontrol analysis of the 'call bell' outbreak" (2). This was in reference to the contaminated call bells, a previously unrecognized reservoir and potential vector. But was it also alluding to a wake-up call for Canada to the emergence of VRE in its hospitals?

In response to the need for more data describing the epidemiology of VRE in Canada, the Canadian Nosocomial Infection Surveillance Program (CNISP), a collaborative effort of the Canadian Hospital Epidemiology Committee (CHEC), a subcommittee of the Canadian Infectious Disease Society, and the Laboratory Centre for Disease Control (LCDC), Health Canada undertook several initiatives including two-point prevalence surveys, establishment of the Passive Reporting Network (PRN) for VRE occurrences in Canada and the VRE Incidence Surveillance Project for determining the incidence rate of VRE in CHEC facilities. What have we learned about VRE since 1997?

Four major phenotypes of VRE are now recognized: van A, van $B$, van $C$ and van $D(3)$. The van A phenotype is characterized by high level resistance to both vancomycin and teicoplanin. It has been found in a number of enterococcal species. The van B phenotype has low to moderate level resistance to vancomycin with preserved teicoplanin susceptibility. It is seen primarily in Enterococcus faecalis and Enterococcus faecium. Van C resistance, found in non-E faecalis and non-Efae- cium, is nontransferrable (constitutive) and exhibits low level resistance to vancomycin. Finally, the van $\mathrm{D}$ phenotype is characterized by low to moderate level resistance to both vancomycin and teicoplanin.

In the United States, data from the National Nosocomial Infection Surveillance System (NNIS) of the Centers for Disease Control and Prevention (CDC), Atlanta, Georgia, have revealed a dramatic national outbreak of VRE. Between 1989 and 1997, the percentage of enterococci isolated from nosocomial infections in intensive care unit (ICU) patients that were resistant to vancomycin rose from $0.4 \%$ to $23.2 \%$ and the percentage from non-ICU patients rose from $0.3 \%$ to $15.4 \%$ (5). From January to December 1999 , VRE accounted for $25.2 \%$ of enterococci associated with nosocomial infections in ICU patients participating in NNIS, a $43 \%$ increase in resistance over the previous five years (5). Rates of vancomycin resistance in enterococci remained lower in non-ICU inpatient areas (11.7\%) and in outpatient areas (3.6\%) (4). Initially found mainly in large hospitals, VRE is now found in American hospitals of all sizes (4).

At the 4th Decennial International Conference on Nosocomial and Healthcare-associated Infections, held March 5 to 9, 2000, a number of American centres described their experience with VRE. Dialysis patients remain a group with increased rates of VRE colonization. Tokars et al (6) found a prevalence of $9.2 \%$ at seven outpatient dialysis centres in Virginia and Maryland where patients were screened over several months in early 1998. During 1997 and 1998, VRE was isolated from $15.6 \%$ of dialysis patients screened within two days of admission to hospital in Atlanta, Georgia (7). For the nine outpatient clinics from where more than 20 patients were cultured in this study, VRE carriage ranged from $10 \%$ to $23 \%$ (7). While adult ICUs have previously been recognized as high prevalence settings for VRE, De Santis et al (8) reported an

\footnotetext{
${ }^{1}$ Queen Elizabeth II Health Sciences Centre and Dalhousie University, Halifax, Nova Scotia; ${ }^{2}$ University Health Network, University of Toronto, Toronto, Ontario

Correspondence and reprints: Dr Lynn Johnston, Room 5014 ACC, Queen Elizabeth II Health Sciences Centre, 1278 Tower Road, Halifax, Nova Scotia B3H 2Y9. Telephone 902-473-8477, fax 902-473-7394, e-mail ljohnsto@is.dal.ca and Dr John Conly, Department of Medicine, Division of Infectious Diseases, Suite 13-118 Norman Urquhart Wing, Toronto General Hospital, University Health Network, 200 Elizabeth Street, Toronto, Ontario M5G 2C4. Telephone 416-340-4858, fax 416-340-5047, e-mail john.conly@uhn.on.ca
} 
outbreak of VRE involving 15 newborns in a level III neonatal ICU in Michigan between August and November 1997. A study completed in Worcester, Massachusetts, found that although VRE cases continued to predominate in the ICUs, cases originating from long term care facilities (approximately $17 \%$ of VRE colonized patients) and wards (23\% of nosocomial acquisitions) were occurring more commonly (9). These data build on previous studies that identified ICU, dialysis, oncology and transplantation patients as those at increased risk of being colonized with VRE $(1,3,10)$.

Prevalence rates in long term care facilities have been more variable. While Bonilla et al (11) found that the prevalence rate of VRE colonization went from $9 \%$ to $22 \%$ in residents of a Michigan long term care facility screened between 1994 and 1996, no colonized patients were found at a similar facility in Atlanta during the same time frame (12).

Despite initially low rates, the potential for transfer of VRE from acute care facilities to long term care facilities exists wherever there is VRE. During early 1997, the Siouxland area in Iowa found dissemination of VRE to multiple long term care facilities as a result of resident transfers from acute care facilities (13). Needless to say, this raises the concern for unrecognized transfer of VRE between facilities and the possibility that long term care facility residents will be a continuing reservoir of infection for acute care facilities.

Worldwide, the epidemiology of VRE is just being defined. Of interest to note is that VRE was first reported from Europe in the 1980s (14). Historically, however, the incidence of clinical VRE infection has been markedly lower there than in the United States (15). A survey of 730 enterococcal strains of clinical significance from 22 microbiology laboratories in Germany found that only $1.5 \%$ were resistant to vancomycin, and all carried the van A gene (16). This is despite early studies from several European countries demonstrating overall colonization rates in hospitalized patients of $1.8 \%$ to $5 \%(17,18)$, among dialysis patients of $15 \%$ (18) and ICU patients of $16.3 \%$ (19). A study of hospitalized hematology patients in a cattlerearing area in the southwest area of France found that $8.6 \%$ of patients were colonized with vancomycin-resistant $E$ faecium, again all of the van A genotype (20). Although five of the nine strains were resistant to ampicillin, none exhibited high level gentamicin resistance (20). Descheemaeker et al (21) recently reported on VRE in Belgian renal dialysis units. of the 1318 patients screened, 14\% were colonized with vancomycin-resistant $E$ faecium or E faecalis, all of the van A genotype. The carriage rate ranged from $0 \%$ to $23.4 \%$ among the various centres. Resistance to ampicillin was found in $10.9 \%$ of strains, and no high level gentamicin resistance was detected. Not all European countries or patient populations have similar prevalence rates of VRE. No vancomycinresistant $E$ faecalis or E faecium were found in 616 patients from seven Norwegian hospitals (22). A study of 338 children and adolescents with end-stage renal failure in Belgium found that only two $(0.6 \%)$ had VRE (23).

In contrast with the low rates of VRE colonization in European patients is the higher rate of VRE colonization found in outpatients and community-based volunteers compared with the United States. The previously mentioned study of hematology patients in southwest France found a VRE carriage rate of $1.8 \%$ in a control population consisting of 169 individuals attending an industrial medicine clinic and hospital employees (20). In Germany, rates of VRE colonization among the nonhospitalized adult population ranged between $0.9 \%$ and $4.2 \%$ (19). Other studies have shown rates varying between $2 \%$ and $12 \%$ in Europe, with isolates being primarily $E$ faecium of the van A genotype $(15,24)$. This is in distinct contrast with the United States where no VRE were found in two studies of community-based individuals $(25,26)$. The presence of VRE in the stools of nonhospitalized patients suggests that it may be acquired in the community.

In Europe, where VRE are also found in domestic farm animals, the resistance pattern and phenotype of human and animal isolates of VRE are similar, with a high prevalence of van A E faecium found in the intestine of animals (15). A recent review of the use of antimicrobial growth promoters in food animals and Efaecium resistance noted the association between use of avoparcin (a glycopeptide) and the presence of VRE in fecal samples collected from the animals of the herds and flocks (27). Finding VRE in the stool of nonhospitalized individuals in a cattle region of France, where avoparcin had been in use until banned in Europe (20), and its absence in the stools of patients from Norway, where avoparcin had never been licensed (22), provides further support to this observation. It has, therefore, been suggested that VRE was introduced into European patients through the food chain and via the community.

Comparison of prevalence data must be made with caution. Rate differences, although potentially representing true ones, may also reflect differences in patient populations studied and laboratory methodologies. Ieven et al (18) have found that the use of an enrichment technique results in a higher recovery of VRE. The culture technique may, therefore, influence the ability to detect VRE that may be present in small quantities in many colonized individuals. While a recent abstract showed no difference in detecting colonized patients with use of nonenrichment techniques (28), the potential for detection error must always be considered when comparing rates from different investigators.

Elsewhere in the world, data on vancomycin resistance in enterococci are also emerging. A national surveillance program to assess bacterial resistance in 27 Argentinean centres found that $2 \%$ of isolates were glycopeptide resistant (29). In a prevalence study of high risk patients in South Africa, 13 of 184 (7\%) individuals were identified as colonized with VRE (30). In a study in Israel, $27 \%$ of ICU patients and $4.8 \%$ of dialysis patients were colonized with VRE, all of the van A phenoytpe, $87.5 \%$ ampicillin resistant and $88 \%$ identified as E faecium (31). Grayson et al (32) found VRE colonization in 12 of $574(2.1 \%)$ patients in a Melbourne, Australia hospital, 11 of whom were dialysis patients. Nine of the isolates were E faecalis (seven van B and two negative for resistance genes) and three Efaecium (all van B). By August of 1998, strains of VRE had been reported from six of eight states or territories of Australia (33). The majority of isolates were van B E faecium 
or Efaecalis. At the Decennial meeting, a group of investigators from Japan (34) showed that VRE were found infrequently (one of 1793 isolates), whereas 28 of 244 (11.5\%) patients hospitalized in Korea were colonized with van A-type VRE (35). Clearly, VRE as clinical pathogens and colonizing bacteria have been identified worldwide.

In Canada, the first prevalence survey for VRE, conducted over a four-week period between January and March 1996, found a rate of $0.1 \%$ among high risk patients in a hospital with no outbreak and 3.7\% among high risk patients in an endemic hospital (36). All but one of the 26 isolates came from Ontario and were of the van B genotype. Twenty-three isolates (88.5\%) were E faecium. Of the VRE patients, $92 \%$ were receiving dialysis. The second prevalence survey, performed in September 1997 , found a rate of only $0.2 \%$ (personal communication, M Ofner-Agostini). To the end of 1998, the CNISP passive reporting network had identified 1315 cases of VRE throughout Canada, with less than $5 \%$ of cases identified as representing infection (38). In a survey of 1487 enterococcal isolates from high risk patients in 12 Canadian tertiary care hospitals between October 1995 and November 1996, 18 (1.2\%) were identified as vancomycin-resistant $E$ faecium (38). Of these, half were van $A$ and half were van $B$, all were ampicillin and ciprofloxacin resistant, and half had high level gentamicin resistance (37). In the first year of data collection for the VRE Incidence Surveillance Program, 95 cases of VRE have been reported to date for a rate of $0.19 / 1000$ patient admissions and representing $0.55 \%$ of enterococcal isolates from the participating facilities (personal communication, L Johnston). Eight of the 93 isolates for which information was available were reported as coming from a clinical infection. Despite the close physical proximity of Canada to the United States, VRE has not attained the same colonization rate and is very rarely encountered as a cause of infection.

In addition to differences in the rates of VRE colonization and infection among Europe, Canada and the United States are differences in the relative frequency of $E$ faecalis and Efaecium as causes of enterococcal infection. While $E$ faecium infections have become increasingly more common in the United States, they remain relatively infrequent in Europe and Canada $(15,16,39)$. Other differences include the in vitro susceptibility of the enterococci. Most VRE in the United States are resistant to other antibiotics such as the fluoroquinolones, ampicillin and gentamicin, whereas those in Europe are more frequently susceptible to these two agents $(15,20,21,40,41)$. Additionally, several studies of the epidemiology of VRE in Europe have shown a high genetic variability among the strains isolated $(15,20,21,41)$. This genetic variability suggests two possible means of occurrence: patients bring their VRE in from the community or there is spread of the enterococcal resistance genes from one strain to another $(20,21)$. On the other hand, many American studies have documented a clonal nature of spread, suggesting that nosocomial transmission is the major mode of transmission $(11,12,15,42)$.

There has been very little new learned about risk factors for colonization or infection with VRE over the past three years. Identified risk factors continue to be prior antibiotic therapy, the number of antibiotics received, the duration of antibiotic therapy, prolonged hospitalization, hospitalization in an ICU, coexistant severe disease and exposure to other colonized patients $(1,3,5,10)$. Our understanding of some of these risk factors has been further refined. It is suggested that antibiotics that particularly affect the anaerobic flora may pose the greatest risk for colonization with VRE (43). Additionally, the risk due to intravenous vancomycin may be a result of hepatobiliary excretion occurring after several days of therapy, indicating that courses exceeding five days should be particularly avoided (44). While the outcomes of patients with bacteremia due to VRE are poor, with crude mortality rates ranging from $37 \%$ to $76 \%$ (45), there have now been several studies which have demonstrated that vancomycin resistance is not an independent predictor of mortality when disease severity is controlled for (45-48).

Studies continue to confirm that environmental contamination is common with VRE $(2,11,37,49,50)$ and that these bacteria may remain viable from days to months (50). Both Noskin et al (50) and Bonilla et al (11) have demonstrated that VRE can be isolated from the hands of health care workers. Contamination is more likely to occur in the environment of patients who are colonized at several sites (49). While the evidence supports a role for environmental contamination in the nosocomial spread of VRE, the relative importance of the human as opposed to the environmental reservoir is not yet known. On the positive side, Rutala et al (51) were able to demonstrate that all cleaning and disinfecting procedures using a moistened cloth were highly effective in removing or inactivating VRE from contaminated surfaces. They emphasized that surface disinfection must involve contact with all contaminated surfaces. They also found that the transfer of VRE from a contaminated surface via gloved hands was poor, suggesting that low level environmental contamination is not likely to play a major role in VRE transmission (51). This supports the use of handwashing and that recommended barrier precautions are indicated. Research efforts should focus on improving the compliance of health care providers with the currently recommended handwashing and barrier precautions (52).

The application of infection control measures to control the spread of VRE has met with variable adherence and variable results. However, several studies have demonstrated the efficacy of CDC recommendations in limiting VRE transmission within acute care facilities (53-56). Such efforts may be most effective in an epidemic situation. One centre found that contact precautions could be eliminated without affecting the rate of clinically significant VRE infection; however, colonization rates were not followed (57). In terms of screening, institution of patient surveillance at the time of transfer from other facilities resulted in a reduction in the rate of rise of the VRE colonization rate at a hospital in Virginia (58). However, another study found that culturing roommates of colonized patients was not helpful in detecting new cases (12). Optimal measures to prevent transmission of VRE in long term care facilities are not known. While Armstrong-Evans et al (59) successfully controlled an outbreak of VRE in a long term care facility after relatively strict infection control measures, Greenaway and 
Miller (60) documented little or no transmission with less stringent measures in a nonoutbreak situation.

Why are there such profound differences in VRE occurrences among the United States, Europe and Canada? Many studies have demonstrated that the prior use of antimicrobials in general, as well as the use of oral vancomycin, are independent risk factors for colonization and infection with VRE. Stool carriage may be prolonged, up to years in some people (5). Colonized patients may serve as a reservoir of organisms, which can be introduced into other facilities or units within the same facility (13). Giving antimicrobials to patients already colonized with VRE may increase the concentration of fecal colonization and the degree of environmental contamination with VRE, especially if patients develop diarrhea as a result of antimicrobial use. Recent data indicate that vancomycin and third-generation cephalosporin use are higher in the United States than in major European centres (5). European VRE typically remain more susceptible to other antibiotics than VRE in the United States (15). Because these isolates are relatively more susceptible and with less selective pressure because of the lower use of vancomycin, it has been suggested that outbreaks are relatively more easy to control. The VRE that are seen in the United States, therefore, have been selected by antibiotic pressure, and, because of broad antimicrobial resistance, have been difficult to eradicate.

We have gained more knowledge about the risks for colonization and infection with VRE and a better understanding of its role in mortality. Our knowledge base remains incomplete for determining the most effective ways of controlling the emergence and spread of VRE. It is clear, however, that antibiotic pressures contribute to its development. While data are scant, there is some literature suggesting that programs designed to limit the use of vancomycin $(61,62)$, cephalosporins and clindamycin (55) result in reduced colonization rates with VRE. Implementation of policies and programs to decrease and improve the use of antimicrobials must be the mainstay of our control efforts. There is evidence that enhanced barrier precautions can limit the transmission if consistently applied in the appropriate setting. Whether they are always required in the acute care setting remains to be determined. The optimal control measures in long term care are as yet uncertain.

\section{REFERENCES}

1. Conly J, Shafran S. Emerging epidemiology of vancomycin-resistant enterococci in Canada. Can J Infect Dis 1997;8:182-5.

2. Lior L, Litt M, Hockin J, et al. Vancomycin-resistant enterococci arrives in Canada: A case-control analysis of the "call bell" outbreak. 36th Interscience Conference on Antimicrobial Agents and Chemotherapy. New Orleans, September 15-18, 1996. (Abst C015)

3. Perl TM. The threat of vancomycin resistance. Am J Med 1999;106:26S-37S.

4. Semiannual Report: Aggregated Data from the National Nosocomial Infections Surveillance System December 1999. Atlanta: Centers for Disease Control and Prevention and United States Department of Health and Human Services, 1999. www.cdc.gov/ncidod/hip/surveill/nnis.htm

5. Martone WJ. Spread of vancomycin-resistant enterococci: why did it happen in the United States? Infect Control Hosp Epidemiol 1998;19:539-45.
6. Tokars JI, Gehr T, Parrish J, Qaiyumi S, Light P. Vancomycin-resistant enterococci colonization at selected outpatient hemodialysis centres. 4th Decennial International Conference on Nosocomial and Healthcare-associated Infections. Atlanta, March 5-9, 2000. (Abst P-T2-56)

7. Steinberg JP, Howard R, Ho T, Maher M, Hackman B, Jernigan J. Vancomycin-resistant enterococcus in dialysis patients: evidence of hospital acquisition. 4th Decennial International Conference on Nosocomial and Healthcare-associated Infections. Atlanta, March 5-9, 2000. (Abst P-T2-57)

8. De Santis LJ, Sturm LK, Thirumoorthi MC, Baran J, Dietrich S. Investigation and control of vancomycin-resistant enterococcus in a level III neonatal intensive care unit. 4th Decennial International Conference on Nosocomial and Healthcare-associated Infections. Atlanta, March 5-9, 2000. (Abst P-T2-58)

9. Lai KK, Fontecchio SA, Kelley AL, Melvin ZS. The changing epidemiology of vancomycin-resistant enterococci. 4th Decennial International Conference on Nosocomial and Healthcare-associated Infections. Atlanta, March 5-9, 2000. (Abst P-T2-62)

10. Boyce JM. Vancomycin-resistant enterococcus. Detection, epidemiology, and control measures. Infect Dis Clin North Am 1997;11:367-84.

11. Bonilla HF, Zervos MA, Lyons MJ, et al. Colonization with vancomycin-resistant Enterococcus faecium: comparison of a long-term care unit with an acute-care hospital. Infect Control Hosp Epidemiol 1997;18:333-9.

12. Tokars JI, Satake S, Rimland D, et al. The prevalence of colonization with vancomycin-resistant Enterococcus at a Veterans' Affairs institution. Infect Control Hosp Epidemiol 1999;20:171-5.

13. Trick WE, Kuehnert MJ, Quirk SB, et al. Regional dissemination of vancomycin-resistant enterococci resulting from interfacility transfer of colonized patients. J Infect Dis 1999;180:391-6.

14. Leclercq R, Derlot E, Duval J, Courvalin P. Plasmid medicated resistance to vancomycin and teicoplanin in Enterococcus faecium. N Engl J Med 1988;319:157-61.

15. Goossens H. Spread of vancomycin-resistant enterococci: differences between the United States and Europe. Infect Control Hosp Epidemiol 1998;19:546-51.

16. Reinert RR, Conrads G, Schlaeger JJ, et al. Survey of antibiotic resistance among enterococci in North-Rhine - Westphalia, Germany. J Clin Microbiol 1999;37:1638-41.

17. Jordens JZ, Bates J, Griffiths DT. Faecal carriage and nosocomial spread of vancomycin-resistant Enterococcus faecium. J Antimicrob Chemother 1994;34:515-28.

18. Ieven M, Vercautern E, Descheemaeker P, Van Laer F, Goossens H. Comparison of direct plating and broth enrichment culture for detection of intestinal colonization by glycopeptide-resistant enterococci among hospitalized patients. J Clin Microbiol 1999;37:1436-40.

19. Wendt C, Krause C, Xander LV, Loffler D, Floss H. Prevalence of colonization with vancomycin-resistant enterococci in various population groups in Berlin, Germany. J Hosp Infect 1999;42:193-200.

20. Gambarotto K, Ploy MC, Turlure P, et al. Prevalence of vancomycin-resistant enterococci in fecal samples from hospitalized patients and non-hospitalized controls in a cattle-rearing area of France. J Clin Microbiol 2000;38:620-4.

21. Descheemaeker P, Ieven $M$, Chapelle $S$, et al. Prevalence and molecular epidemiology of glycopeptide-resistant enterococci in Belgian renal dialysis units. J Infect Dis 2000;181:235-40.

22. Simonsen GS, Anderson BM, Digranes A, et al. Low faecal carrier rate of vancomycin-resistant enterococci in Norwegian hospital patients. Scand J Infect Dis 1998;30:465-8.

23. Von Baum H, Schehl J, Geiss HK, Schaefer F and the Mid-European Pediatric Peritoneal Dialysis Study Group. Prevalence of vancomycin-resistant enterococci among children with end-stage renal failure. Clin Infect Dis 1999;29:912-6.

24. Endtz HP, van den Braak N, van Belkum A, et al. Fecal carriage of vancomycin-resistant enterococci in hospitalized patients and those living in the community in the Netherlands. J Clin Microbiol 1997;35:3026-31.

25. Coque TM, Tomayko JF, Ricke SC, Okhyusen PC, Murray BE. Vancomycin-resistant enterococci from nosocomial, community, 
and animal sources in the United States. Antimicrob Agents Chemother 1996;40:2605-9.

26. Silverman J, Thal LA, Perri MB, Bostic G, Zervos MJ. Epidemiologic evaluation of antimicrobial resistance in community-acquired enterococci. J Clin Microbiol 1998;36:830-2.

27. Wegener HC, Aarestrup FM, Jensen LB, Hammerum AM, Bager F. Use of antimicrobial growth promoters in food animals and Enterococcus faecium resistance to therapeutic antimicrobial drugs in Europe. Emerg Infect Dis 1999;5:329-35.

28. Reisner BS, Shaw S, Huber ME, et al. Comparison of broth enrichment and direct inoculation of solid media for recovery of vancomycin-resistant enterococci from perirectal and environmental surface samples. 4th Decennial International Conference on Nosocomial and Healthcare-associated Infections. Atlanta, March 5-9, 2000. (Abst P-T2-50)

29. Bantar C, Famiglietti A, Goldberg M. Three-year surveillance study of nosocomial bacterial resistance in Argentina. Int J Infect Dis 2000;4:85-90.

30. von Gottberg A, von Nierox W, Duse A, et al. Epidemiology of glycopeptide-resistant enterococci colonizing high-risk patients in hospitals in Johannesburg, Republic of South Africa. J Clin Microbiol 2000;38:905-9.

31. Dan M, Poch F, Leibson L, Smetana S, Priel I. Rectal colonization with vancomycin-resistant enterococci among high-risk patients in an Israeli hospital. J Hosp Infect 1999;43:231-8.

32. Grayson ML, Grabach EA, Johnson PD, et al. Outcome of a screening program for vancomycin-resistant enterococci in a hospital in Victoria. Med J Aust 1999;171:133-6.

33. Bell J, Turnidge J, Coombs G, O'Brien F. Emergence and epidemiology of vancomycin-resistant enterococci in Australia. Commun Dis Intell 1998;22:249-52.

34. Fujita N, Komori T, Yoshimura M, Tanimoto K, Ike Y. The fecal carriage of vancomycin-resistant enterococci in in- and outpatients and healthy people in Kyoto, Japan. 4th Decennial International Conference on Nosocomial and Healthcare-associated Infections. Atlanta, March 5-9, 2000. (Abst P-T2-54)

35. Choi SJ, Kim DR, Cheong HJ, Kim WJ, Kim MJ, Park SC. Rectal surveillance and control of vancomycin-resistant enterococci colonization in intensive care unit at one Korean univ hospital. 4th Decennial International Conference on Nosocomial and Healthcare-associated Infections. Atlanta, March 5-9, 2000. (Abst P-T2-55)

36. Ofner-Agostini ME, Conly J, Paton S, et al. Vancomycin-resistant enterococci (VRE) in Canada - Results of the Canadian Nosocomial Infection Surveillance Program 1996 VRE point prevalence surveillance project. Can J Infect Dis 1997;8:73-8.

37. Conly JM, Ofner ME, Paton S, et al. The emerging epidemiology of vancomycin-resistant enterococci in Canada 1993-1998: Results from the Canadian Nosocomial Infection Surveillance Program (CNISP) Passive Reporting Network. 67th Conjoint Meeting on Infectious Diseases. Edmonton, October 31 to November 4, 1999. Can J Infect Dis 1999;10:38D. (Abst G5)

38. Karlowsky JA, Zhanel GG, Haban DJ. Vancomycin-resistant enterococci (VRE) colonization of high-risk patients in tertiary care Canadian hospitals. Canadian VRE Surveillance Group. Diagn Microbiol Infect Dis 1999;35:1-7.

39. Madani TAA, Kabani A, Orr P, Nicolle L. Enterococcal bacteremia in a tertiary care centre in Winnipeg. Can J Infect Dis 1999; 10:57-63.

40. Morris JG, Shay DK, Hebdan JN, et al. Enterococci resistant to multiple antimicrobial agents, including vancomycin. Establishment of endemicity in a university medical centre. Ann Intern Med 1995;123:250-9.

41. Vandamme P, Vercauteren E, Lammens C, et al. Survey of enterococcal susceptibility patterns in Belgium. J Clin Microbiol 1996;34:2572-6.

42. Moreno F, Grota P, Grigs C, et al. Clinical and molecular epidemiology of vancomycin-resistant Enterococcus faecium during its emergence in a city in southern Texas. Clin Infect Dis 1995;21:1234-7.

43. Donskey CJ, Hanrahan JA, Hutton RA, Rice LB. Effect of parenteral antibiotic administration on persistance of vancomycin-resistant Enterococcus faecium in the mouse gastrointestinal tract. J Infect Dis 1999;180:384-90.
44. Lemos-Filho, Currie BP. Evidence that parenteral vancomycin therapy can act as a selective pressure on human bowel flora. 4th Decennial International Conference on Nosocomial and Healthcare-associated Infections. Atlanta, March 5-9, 2000. (Abst S-W4-04)

45. Garbutt JM, Ventrapragada M, Littenburg B, Mundy LM. Association between resistance to vancomycin and death in cases of Enterococcus faecium bacteremia. Clin Infect Dis 2000;30:466-72.

46. Lucas GM, Lechtzin N, Puryear W, Yau LL, Flexner CW, Moore RD. Vancomycin-resistant and vancomycin-susceptible enterococcal bacteremia: comparison of clinical features and outcomes. Clin Infect Dis 1998;26:1127-33.

47. Lautenbach E, Bilker WB, Brennan PJ. Enterococcal bacteremia: risk factors for vancomycin resistance and predictors of mortality. Infect Control Hosp Epidemiol 1999;20:318-23.

48. Stroud L, Edwards J, Danzig L, Culver D, Gaynes R. Risk factors for mortality with enterococcal bloodstream infections. Infect Control Hosp Epidemiol 1996;17:576-80.

49. Bonten MJM, Hayden MK, Nathan C, et al. Epidemiology of colonization of patients and environment with vancomycin-resistant enterococci. Lancet 1996;348:1615-9.

50. Noskin GA, Stosor V, Cooper I, Peterson LR. Recovery of vancomycin-resistant enterococci on fingertips and environmental surfaces. Infect Control Hosp Epidemiol 1995; 16:577-81

51. Rutala WA, Weber DJ, Gergen MF. Vancomycin-resistant enterococcus sp surface disinfection and transmissibility via contaminated surfaces. 4th Decennial International Conference on Nosocomial and Healthcare-associated Infections. Atlanta, March 5-9, 2000. (Abst P-T2-72)

52. Weber DJ, Rutala WA. Role of environmental contamination in the transmission of vancomycin-resistant enterococci. Infect Control Hosp Epidemiol 1997;18:306-9.

53. Montecalvo MA, Jarvis WR, Uman J, et al. Infection control measures reduce transmission of vancomycin-resistant enterococci in an endemic setting. Ann Intern Med 1999; 131:269-72.

54. Jochimsen EM, Fish L, Manning K, et al. Control of vancomycin-resistant enterococci at a community hospital efficacy of patient and staff cohorting. Infect Control Hosp Epidemiol 1999;20:106-9.

55. Ouale J, Landman D, Saurina G, Atwood E, DiTore V, Patel K. Manipulation of a hospital antimicrobial formulary to control an outbreak of vancomycin-resistant enterococci. Clin Infect Dis 1996;23:1020-5

56. Thompson RL, Marshall WF, Maker MD, et al. Control of vancomycin-resistant enterococcus in a transplant program. 4th Decennial International Conference on Nosocomial and Healthcare-associated Infections. Atlanta, March 5-9, 2000. (Abst P-T2-67)

57. Oda GV, DeVries MM, Valdon CA, Higby LA, von Husen M, Loutit JS. No change in vancomycin-resistant enterococcus rates after elimination of contact precautions at a Veterans Affairs Medical Centre. 4th Decennial International Conference on Nosocomial and Healthcare-associated Infections. Atlanta, March 5-9, 2000. (Abst P-T2-71)

58. Calfee DP, Giannetta ET, Durbin LJ, Farr BM. Control of vancomycin-resistant enterococcus colonization among inpatients at a tertiary care facility. 4th Decennial International Conference on Nosocomial and Healthcare-associated Infections. Atlanta, March 5-9, 2000. (Abst P-T2-69)

59. Armstrong-Evans M, Litt M, McArthur MA, et al. Control of transmission of vancomycin-resistant Enterococcus faecium in a long-term care facility. Infect Control Hosp Epidemiol $1999 ; 20: 312-7$

60. Greenaway CA, Miller MA. Lack of transmission of vancomycin-resistant enterococci in three long-term care facilities. Infect Control Hosp Epidemiol 1999;20:341-3.

61. Anglim AM, Klym B, Byers KE, Scheld WM, Farr BM. Effect of a vancomycin restriction policy on ordering practices during an outbreak of vancomycin-resistant Enterococcus faecalis. Arch Intern Med 1997;157:1132-6.

62. Burke CE, Piper J, Holloway W. Order form for restricting vancomycin prescribing. Am J Health Syst Pharm 1997;54:1893-7. 


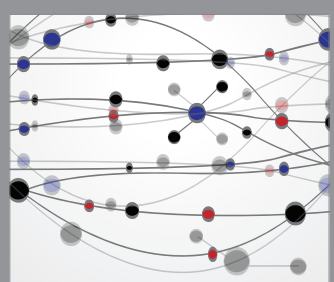

The Scientific World Journal
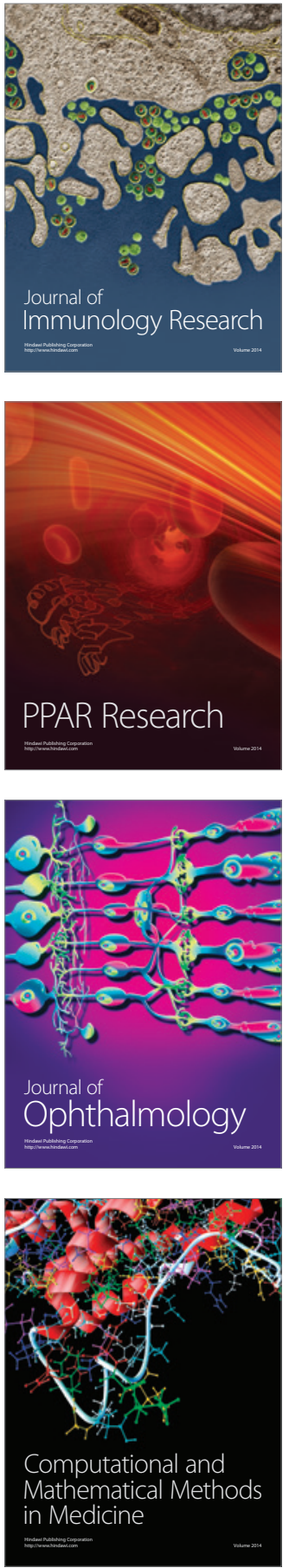

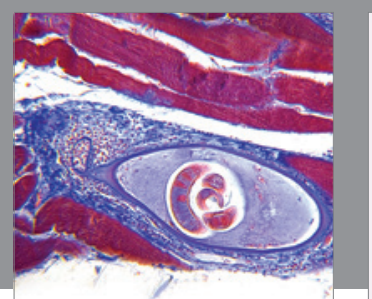

Gastroenterology Research and Practice

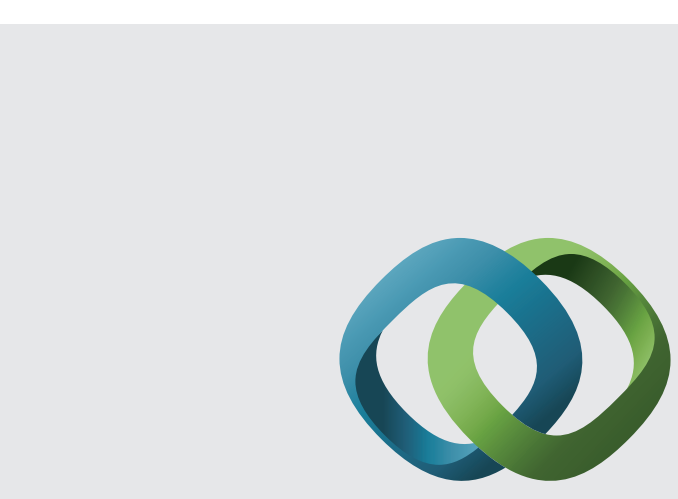

\section{Hindawi}

Submit your manuscripts at

http://www.hindawi.com
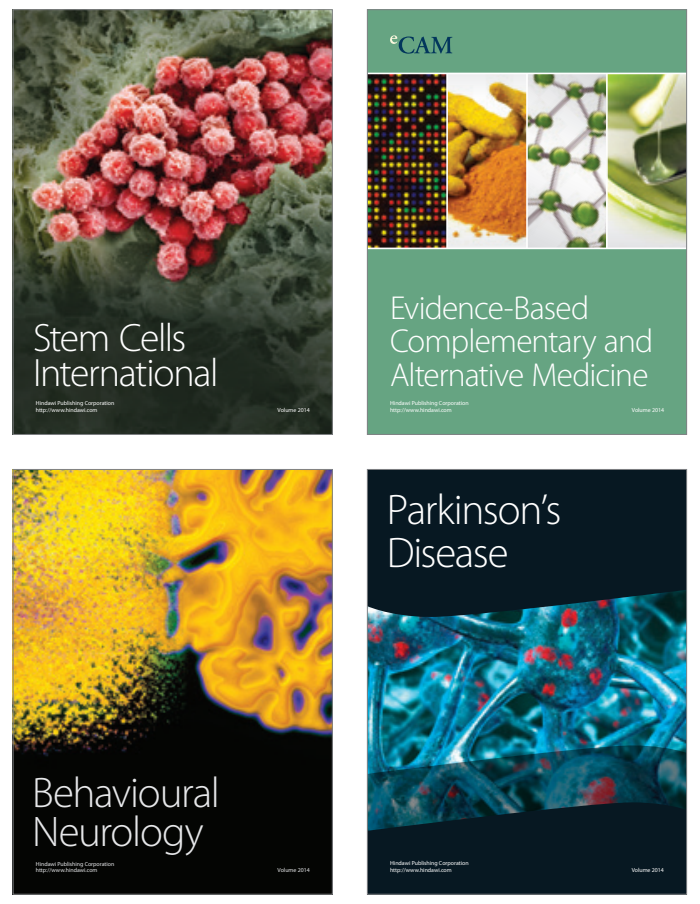
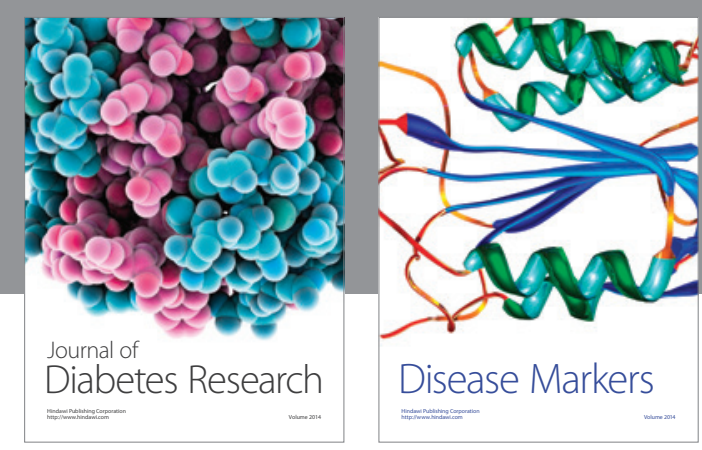

Disease Markers
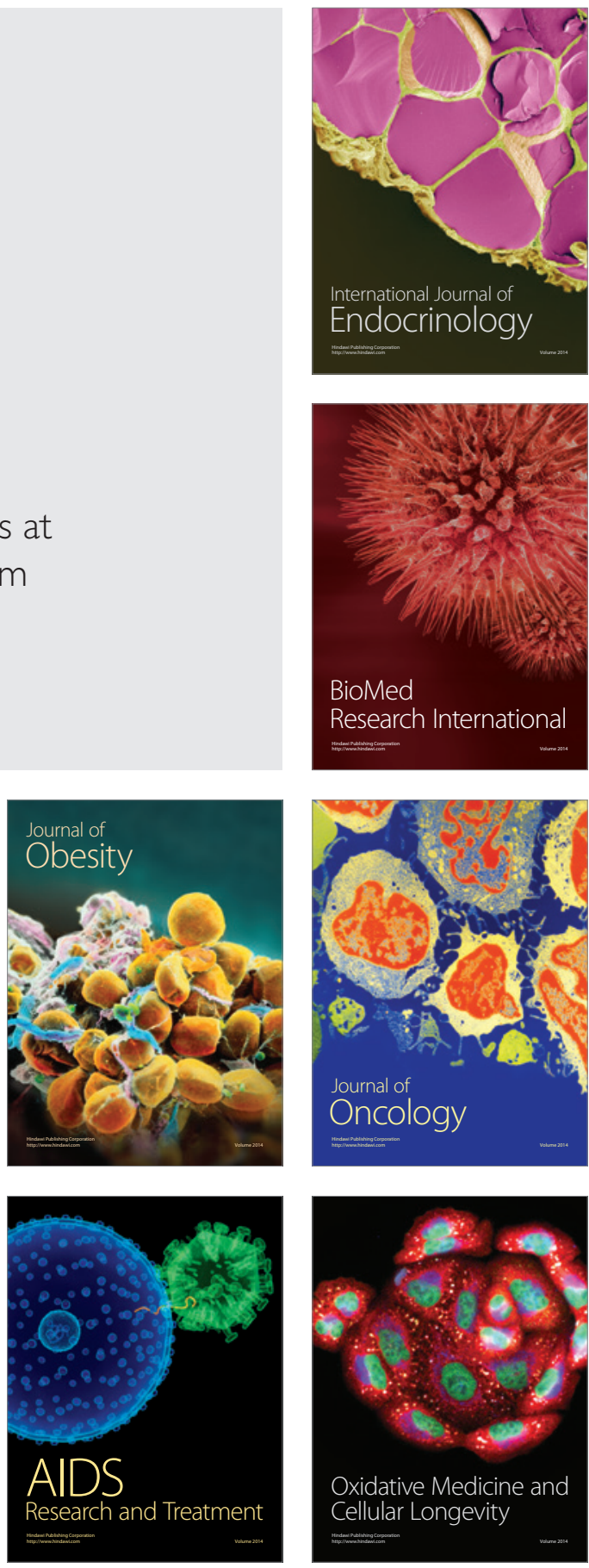